\title{
Lunar Surface Scenarios: Habitation and Life Support Systems for a Pressurized Rover
}

\author{
Molly Anderson ${ }^{1}$, Dr. Anthony Hanford ${ }^{2}$, Dr. Robert Howard ${ }^{3}$, and Larry Toups ${ }^{4}$ \\ ${ }^{1}$ NASA Johnson Space Center, Mailcode EC2, 2101 NASA Parkway, Houston TX, 77058 \\ ${ }^{2}$ ESC Group, Jacobs Sverdrup, Mail Code JE-5EA, PO Box 58447, Houston, TX, 77258 \\ ${ }^{3}$ NASA Johnson Space Center, Mailcode SF3, 2101 NASA Parkway, Houston TX, 77058 \\ ${ }^{4}$ NASA Johnson Space Center, Mailcode EX2, 2101 NASA Parkway, Houston TX, 77058
}

\begin{abstract}
Pressurized rovers will be a critical component of successful lunar exploration to enable safe investigation of sites distant from the outpost location. A pressurized rover is a complex system with the same functions as any other crewed vehicle. Designs for a pressurized rover need to take into account significant constraints, a multitude of tasks to be performed inside and out, and the complexity of life support systems to support the crew. In future studies, pressurized rovers should be given the same level of consideration as any other vehicle occupied by the crew.
\end{abstract}

\section{The Need for Pressurized Rovers}

Explorers throughout history survived using only the necessities that they carried with them. Placing this requirement on an exploring astronaut is a more significant challenge because even elements such as atmosphere, radiation protection, and thermal management must be carried. Pressurized rovers are an enabling technology for future missions to planetary bodies beyond the Earth because they provide the necessities of life for an exploring crew and allow them to more efficiently explore. Rovers also enable mobility so that explorers are not limited to a short radius around an outpost as the only locations available for investigation. This paper examines the major systems for a pressurized rover and outlines the requirements or design considerations that will drive design of the vehicle.

Mobility, radiation protection, and extravehicular activity (EVA) drive the need for a pressurized rover. Crews on the lunar surface may need to travel to locations between $75 \mathrm{~km}$ and to $200 \mathrm{~km}$ from an outpost to support prescribed exploration science investigations. Specific sites of interest may have exposed layers of rock or regolith due to meteor activity, or thermal extremes such as persistent cold that preserves ice. These characteristics are generally not shared with viable locations for a lunar outpost, which requires level terrain to assemble elements and land and launch spacecraft, all with easy traverses in between and a relatively benign thermal climate. 
With current and proposed spacesuit technology, astronauts cannot walk for 200 $\mathrm{km}$ to exploration sites, so rover assistance is necessary. Based on Apollo technology and current Earth examples (DARPA, 2005), $20 \mathrm{~km} / \mathrm{hr}$ is a reasonable speed for a pressurized rover, and a $200 \mathrm{~km}$ journey would require ten hours, which is too long to leave an astronaut to rely on a spacesuit alone.

Another critical driver is radiation protection. Intense solar flares and storms can occur with less than an hour of warning time (Phillips, 2000). The crew would be unable to return to the outpost for protection quickly enough. As a result, a crew must take a radiation shelter with them when traveling away from the outpost.

Other EVA requirements drive the need for a pressurized rover as well. In a gravity field, a crewmember can carry life support consumables for a limited duration, and recharge capabilities need to be available. A pressurized rover may improve dust mitigation efforts in the outpost. Pressurized rovers can also perform duties like unpressurized rovers, such as carrying equipment to a site and returning with samples.

\section{Universal Design Drivers}

One important design driver is the concept of operations for the rover. It is assumed that the pressurized rover will support up to four crewmembers, with a nominal crew size of two to allow rescue scenarios. The rover sortie duration is up to five days. This estimate includes travel out $200 \mathrm{~km}$, up to three EVA days, and a $200 \mathrm{~km}$ return trip.

Several design goals are common for all the rover systems and throughout spacecraft design. Mass, equipment volume, power, heat generated, and crew time required should be minimized. Design for continuous movement over rugged terrain, however, is not something that is not usually considered, but will be necessary here.

\section{Systems Driven by Crew Internal Tasks}

The habitable pressurized volume design will be driven by the required tasks that the rover crew performs. Proper allocation and combination of tasks can allow the effective task execution within constraints of the proposed lunar transportation architecture. After consideration of all of the tasks, an overall internal architecture emerged as a design solution to manage all the tasks, which is similar to previous studies as well (Eckart, 1999 and Joosten, 1994). The rover will consist of three basic sections: a Forward Section, Hygiene Section, and Entry Section. The Forward Section is the primary occupied volume of the rover, containing command and control equipment and seating with restraint harnesses for four crew members, including two Drivers and two Specialists. The Entry Section, at the rear of the rover, contains four suit locks (Cohen 1995), the rover docking interface, and a suit maintenance work area.

The majority of the vessel will be group workspaces. The Forward Section of the rover will serve as the primary location for the crew while the rover is in transit and for certain tasks while the rover is on location. The Entry Section of the rover will facilitate spacesuit ingress/egress, suit maintenance, and access to the lunar outpost.

Driver stations will have access to all vehicle control functions, and display and input devices to be used for driving, navigation, and course plotting. Limited controls should be available at other locations. Hand controllers in the Entry Section will be used for fine positioning during docking. Vehicle systems can be accessed from computers at all stations. Portable computer systems can be utilized to perform tasks related to the EVA mission.

Crew health is an important focus, especially for an EVA tasks. The Health Monitoring System and other medical stowage will be located within reach of the right 
Specialist station. The level of medical care provided on the pressurized rover will be comparable to that provided on the Crew Exploration Vehicle and Lunar Surface Access Module. An injured crew member can be restrained on a reclined seat at a Specialist station that can interface with physiological monitoring equipment and can support medical teleconferences. There will not be a dedicated exercise volume in the rover, but the crew could use stowed equipment such as portable cycle pedals or resistance bands.

The Forward Section will be the location for all meal functions. A stowable table will be used for crew meals, placed between the four seats, which will be able to rotate away from the workstations to face the table. Meal preparation will be conducted at the left Specialist station, with food stowage, a food warmer, and hot and cold water dispensers located within reach. Food stowage will consist of individual servings, sealed before launch to ensure shelf life, stored in non-refrigerated lockers for volume and power savings. Meal cleanup will involve disposal of meal waste products to ensure safety and wiping and stowage of the dining table. Given the short duration of a rover mission, disposable containers and utensils are conceivable, assuming adequate resupply at the outpost. Alternately, some form of wipes and sterilization could be used to clean utensils and dishes.

Crewmembers on the rover will perform housekeeping tasks, including dust mitigation, cleaning and disinfecting surfaces and managing stowage and trash. No maintenance should be required away from the habitat other than basic spacesuit cleaning. Dust mitigation efforts manage the lunar regolith that will inevitably enter the crewed areas. It is likely to be more challenging on a pressurized rover than in a habitat. The crew will perform many EVAs and the dust will accumulate quickly in the small volume. Several strategies can be employed to attempt to manage the issues. The airlock and docking interface designs should minimize the amount of dust that enters the pressurized rover. Filtering techniques help manage dust that does enter. In a gravity field, wiping dust from surfaces and floors could have significant impact. Finally, all technologies and systems in the pressurized rover must be robust and able to operate assuming that some lunar regolith will enter the pressurized crew areas.

Group Recreation will not receive a dedicated volume but will share areas such as the volume used for eating. The vehicle's display devices may also be usable for recreational activities such as watching movies.

Private Workspace will be extremely limited onboard the rover, and primarily confined to hygiene functions, or activities that can be carried into a hygiene volume. Some degree of visual privacy will be provided on individual work stations, due to the positioning of crewmembers and auditory privacy via headphones. It is possible to achieve visual privacy for short durations with one person in the Forward Section, one in the Entry Section, one in the lavatory, and one in the wash room, with the lavatory and wash room doors closed.

Due to the limited volume of the rover, personal spaces will be limited in both volume and privacy. Specific personal spaces are required for tasks such as sleeping, dressing, communication, and recreation. To the extent possible, these tasks will be performed in the volume used for seating, or in a shared-use volume. Sleeping can be accomplished via reclining seats or deployable hammocks. Dedicated sleep bunks will not be provided due to volume constraints. A possible design solution uses hammocks deployed in the Entry Section or Hygiene corridor based on crew preference for crewmembers using Driver seats. Specialist stations will recline to convert seats to a "bed" configuration. Personal communication, including video, voice, and data communication will be conducted from the crewmember's seat. For private 
communications, such as medical conferences or family discussions, the crewmember may choose to enter the hygiene compartment to gain a measure of visual and auditory privacy. Dedicated volume will not be provided for personal recreation, which will essentially consist of computer-based activities (e.g. DVD movies, video games, etc.) and small personal items brought by the crew or crafted on the lunar surface. These activities will be conducted in the crewmember's seat, or other available location.

The Hygiene Section must balance crew privacy with minimal space. Personal hygiene will require a dedicated, shared-use volume. Hygiene tasks include body cleansing, shaving, grooming, oral hygiene, hand/face wash, and urination/defecation. The hygiene compartment will contain two volumes for this purpose, a lavatory and a washroom. Doors can isolate the lavatory and washroom into individual private volumes, leaving a corridor between the Forward Section and Entry Section, or into a shared volume combining all three components of the hygiene section but blocking access from the Forward Section to the Entry Section. The lavatory will be slightly smaller in volume to a restroom aboard a passenger jetliner and will not include a sink. The washroom will be similar in size. It will provide volume for shaving, grooming, oral hygiene, body cleansing, and hand/face wash. It remains an open issue whether hygiene water can be included in the rover. If water is provided, then lavatory hygiene functions can be much like on Earth. However, it could prove difficult to transport sufficient water. Wipes for cleansing are becoming increasingly popular in commercial use (e.g. hand or face wash wipes), but may suffer limitations for tasks such as removing grit from underneath fingernails. Human waste products will be removed from the hygiene section as their collection bags fill, nominally between rover sorties. Upon return to the outpost they will be delivered to the outpost's waste containment facility. In a contingency, waste bags can be removed during a sortie and relocated to internal stowage or the rover exterior. Crewmembers may choose to dress in any of the open areas throughout the rover, or in the lavatory or wash room, allowing a measure of privacy.

\section{Life Support Systems}

While developing a vehicle to traverse the moon reliably is challenging in itself, maintaining a viable environment for human beings is a primary design consideration. Supporting human metabolic loads, such as the evolution of carbon dioxide and trace gases, urine and feces, and consumption of oxygen, water, and food, becomes a design criterion. Nominal loads may be defined by assuming a generic human with nominal intakes and outputs. Human beings, however, vary widely and their metabolic loads also vary. Thus, any design must accommodate human variability. The overall metabolic load may vary during a mission from one individual to the maximum permissible crew size. Some crew metabolic loads are stipulated via requirements, such as HSIS (2005). Other metabolic loads, such as metabolic waste products, arise as the natural result of other decisions and are determined via mass balances. See Hanford (2004).

Though the crew will be away from the rover during EVAs, rover stores are the most likely source of consumables for spacesuits, so the rover's metabolic stores must also consider EVA loads. Because EVA is generally more strenuous than comparable time spent within the rover, metabolic stores for EVA will be larger than for a comparable time in the rover, as outlined in requirements and design guidance.

The air system design addresses several competing issues of great importance to overall crew safety. If the corresponding spacesuits for this rover operate at low-pressure with a pure oxygen atmosphere, then a low-pressure, high-oxygen-content rover atmosphere reduces the necessary pre-breath time to minimize the chance of 
decompression sickness. Correspondingly, higher oxygen content increases the combustibility of most common structural materials, which decreases the time available for the crew to react in the event of a fire. See detail within Lange (2005).

The overall rover pressure design should also consider several physical issues associated with cabin atmospheres. The overall pressure regulation may support varying atmospheric pressures to allow the rover to dock with the outpost, which may operate at a higher pressure than the rover. If so, the rover must accommodate the higher outpost pressure during docking operations. Overall pressure directly affects cooling within the crew cabin. At lower pressures, it is more difficult to remove thermal loads from aircooled hardware and free-standing crew, thus requiring higher velocity fans to move the thinner gases to provide adequate cooling. Further, a lower cabin pressure encourages using coldplates instead of air-cooling for all fixed hardware. (Actually, to be vacuum compatible, fixed hardware should be on coldplates.) Sound travels more slowly in thin atmospheres, impairing verbal communications. While aides for hearing might offset the effects of the thinner gas, this approach could fail in an emergency just because crewmembers are not be wearing their "hearing devices."

Atmosphere composition is another design consideration. Overall pressure dictates the partial pressure of any diluent gases, and this affects the overall cost for atmospheric gases. Oxygen may come either as a commodity from Earth, or it might be gathered locally as an in-situ resource. According to Smith and West (1982), the regolith on the moon is over $60 \%$ oxygen on an elemental basis. Diluent gases are much scarcer. There are minuscule quantities of noble gases on the moon, and little, if any, nitrogen. Thus, nitrogen from Earth is the most likely diluent gas. Further, nitrogen is familiar to doctors, so its use should not require additional research.

The air system monitors and controls trace airborne contaminants, including trace gases and particulate loads. Due to the short duration of rover sorties, some airborne contaminants may not accumulate in sufficient quantities to exceed safety requirements, especially if the rover atmosphere can be renewed between sorties. However, this thesis should be thoroughly analyzed and tested before flight.

Humidity control is a comfort function provided as part of the air system or the thermal system, and can also prevent hazards caused by condensation. Humidity may be removed by two approaches. One removes both sensible and latent thermal loads, via a condensing heat exchanger, and gathers heat and water. This sets the minimum thermal transport loop temperature and dictates heat rejection sizing. A second approach uses carbon dioxide removal technology, such as an amine, to extract and reject both carbon dioxide and water. These latter systems are regenerated by venting to vacuum. For a short-duration vehicle, the second approach is appealing, but it expends more commodities.

Finally fire suppression agents should be non-toxic and compatible with other rover components. Ideally, recovery from fire would have a minimal impact.

From a life support perspective, the food system must be nutritious, appetizing, and safe. The food's primary function provides the crew with a metabolic energy source and all chemical constituents necessary for crew health. Because crewmembers can choose not to eat the food provided, food must be appetizing both to encourage timely consumption and to fulfill the psychological expectations associated with eating. Finally, the food system must be safe.

Active thermal control collects, transports, and rejects thermal loads from the rover. Most design decisions associated with thermal control concern collecting heat to maintain comfortable temperatures for the crew and safe touch temperatures at the 
equipment. Thermal loads leave hardware via the thermal transport fluid loop plus leakage to the cabin via conduction through the structure, convection to the cabin atmosphere off exposed panels, or radiant exchange with other cabin surfaces. As the cabin atmosphere thins, convection decreases. To maintain surface temperatures, more heat must be carried by the thermal transport fluid loop or the temperature will rise. Heat loads are collected either via a gas-liquid heat exchanger or a coldplate in direct physical contact with the hardware. Gas-liquid heat exchangers remove heat from the cabin atmosphere to cool the crew or portable hardware, but collect heat less efficiently than coldplates. At reduced pressures, the gas-phase thermal resistance increases, decreasing the heat removal flux. Fixed hardware may also be cooled by coldplates. Coldplates remove heat loads by conduction and require mounting heat-load-generating hardware on surfaces that are integrated with thermal loop plumbing. The thermal fluid loop can be either a single-fluid, or a dual-loop approach.

Finally, rejection of thermal loads on the moon can be challenging, especially from a rover. Here, mechanisms to reject thermal loads include radiant transfer or releasing heat with a consumable via evaporation or sublimation. Radiant transfer requires sufficient surface area that views a relatively cold environment. Ideally, radiators covering only the rover external hull that is not used for other purposes could provide sufficient radiant surface area for all thermal loads generated, though radiant surface can be increased with deployable radiator panels. Consumables can also be used either in a primary role or in a secondary or supplemental role for cooling.

The waste system accommodates both waste storage and waste transfer operations. Storage accommodates human metabolic wastes, such as urine and feces, trash, such as food packaging, and finally any wastes that are not either human metabolic wastes or trash, such as expended hardware replaceable units. Wastes may be stored together or separately, but must be contained away from the crew. Wastes must also be sufficiently stable for the duration of the rover sortie, but they do not need to be indefinitely stable. Ultimately, the wastes must be removed at the conclusion of the rover sortie, and they may be processed further at the outpost.

The water system design considers supplying potable water, collecting wastewater, such as humidity condensate, hygiene wastewater, or urine, and transferring wastewater to the outpost once the rover sortie ends. Potable water may be supplied from stores because water processing is seldom cost effective for very short duration missions. However, some water polishing steps may assure the quality of water taken from stores before dispensing it to the crew. Wastewater may be source-separated or stored together. Which is used depends on wastewater quantity and how the wastewater is treated after it is transferred to the outpost. Depending upon the carbon dioxide technology selected, humidity condensate may not exist. Likewise, depending on the hygiene approach, whether wipes or free water is employed, hygiene wastewater may not exist.

\section{Systems Driven by Crew External Tasks}

The concept of operations for a pressurized rover has to include both crew inside the vehicle, and activities while the crew is outside the vehicle or inside the outpost, and all the transitions that come between. Experiment deployment and sample collection are likely to be common tasks for an exploring crew. A likely solution is a combination glove-box and airlock concept that would allow the crew to stow samples easily while outside, but also access them while inside for preliminary analysis. Vehicle maintenance is also a critical interface between the rover and the crew. Providing a pressurized garage space would be very expensive, so maintenance will be done during an EVA. All repairs 
must be accomplishable with the limited mobility and dexterity associated with a spacesuit. The commonly replaced items must be extremely easy to access. Any high risk failures must be easy to access, because the useful life of the vehicle ends with any failure that cannot be repaired.

\section{Systems Driven by Interfaces}

The pressurized rover has a relatively complicated operations concept, and interfaces with the crew, a second rover, or the outpost.

The most obvious interface between the crew and the rover is the exit to the surface for EVA. Limited mass and volume for mobility, and significant dust mitigation requirements (Connors, 1994) make the Suitlock architecture (Cohen 1995) a promising solution. With this design, the spacesuit never enters the pressurized rover, which aids dust mitigation. Assuming only a single surface suit per crewmember, before departing in the pressurized rover, the crew must walk in spacesuits out of the outpost to the pressurized rover and dock with the Suitlock to enter the vehicle. The movement of the rover requires that the suits be held above the ground to protect them, so a deployable staircase or other method must be available for the crew to safely reach the surface below. Also, the suits must be protected from lunar regolith dust or debris disturbed by the movement of the rover.

The next critical interface is the vehicle-to-vehicle interface. With limited volume and surface area, a single interface must be able to dock with both the outpost, and another pressurized rover for rescue or joint operations. The crew should be able to pass through this passage while in a spacesuit for emergency egress, fulfilling the requirement for multiple entrances and exits. The complexity and difficulty of docking the pressurized rover will be largely determined by the way this interface is designed. An architecture with flexibility in the path created between the two vehicles could be very helpful to crew members controlling the pressurized rover.

Umbilical lines are required to pass fluids, power, and other resources to and from the vehicle. One umbilical will be needed when the rover is docked to an outpost to allow the crew members in the rover to rely on the outpost's life support. A second umbilical is required to provide consumables to a crew member performing an EVA. The crew will need to periodically attach the portable life support systems (PLSS) to the vehicle and recharge consumables. There is risk in opening a flow path into the life support system while a crew member is on EVA (Connors 1994). Designing to prevent spacesuit depressurization will be a driver in any recharge umbilical system.

The last set of interface issues considered is operational issues. The pressurized rover will provide additional habitable volume to the outpost when docked. Between missions the space could be used as stowage volume, as a conference area, or as extra sleeping area if there are crew change-outs with overlaps. The rover could also operate as a pressure chamber for treatment of decompression sickness. It could also fulfill fault tolerance requirements for separate pressurized volume that can support the crew available in the event of an emergency.

\section{Systems Driven by Mechanical or Engineering Requirements}

Power and energy, while essential, present a series of significant implicit requirements. The power generation approach or energy storage device must be compatible with and not inhibit the rover's mobility, as surface mobility is a primary function. To support mobility, the power availability must be invariant when the vehicle enters a shadow or the Sun position relative to the horizon changes. Finally, because heat 
rejection from the rover will be limited, the parasitic thermal load associated with power generation and transmission should be minimized.

The pressure shell provides several important functions. It contains the cabin atmosphere and provides structure. Additionally, the pressure shell design affects the radiation protection for the vehicle interior and its component materials affect the potential flame propagation rate in the event of a fire. As the overall rover design must be highly integrated, each of these issues should be considered.

\section{Conclusions}

A pressurized rover is a critical technology for enabling effective lunar exploration, but even with a small size, it has a level of complexity similar to other crewed spacecraft. Many design drivers and possible solutions can be established by considering what functions the rover systems must fulfill. In future studies to consider the surface system elements necessary for lunar exploration, the pressurized rover should be given independent attention equivalent to other major systems.

\section{References}

Cohen, M. M. (1995) “The Suitport's Progress,” AIAA-95-1062, AIAA Space Medicine and Life Science Conference.

Cohen, M. M. (2000) “Pressurized Rover Airlocks,” SAE 2000-01-2389. 30th International Conference on Environmental Systems.

Connors, M, Eppler, D, and Morrow, D. (1994) "Interviews with the Apollo Lunar Surface Astronauts in Support of Planning for EVA Systems Design,” NASATM-108846, NASA.

Eckart, P. (1999) The Lunar Base Handbook, The McGraw-Hill Companies, Inc.

Hanford, A. J. (2004) “Advanced Life Support Baseline Values and Assumptions Document,” NASA/CR-2004-208941, NASA.

HSIS (2005) NASA-STD-3000, Volume VIII, NASA, Draft as of 01 August 2005.

Joosten, K., and Guerra, L. (1993) "Enabling Lunar Exploration through Early Resource Utilization,” AIAA Space 1994 Conference.

Lange, K. E. (2005) "Bounding the Spacecraft Atmosphere Design Space for Future Exploration Missions,” LMSEAT 34373, Lockheed Martin Space Operations.

Phillips, T. (2000) "Space Radiation Storm,” http://science.nasa.gov/headlines/y2000/ast14jul_2m.htm

Schoppa, W. (1997) “ITCS Fluids White Paper,” HDID-A44B-827, Lockheed Martin Space Mission Systems and Services.

Smith, R. E., and West, G. S. (1982) "Space and Planetary Criteria Guidelines for Use in Space Vehicle Development, 1982 Revision,” NASA TM 82478, Volume 1, NASA.

DARPA (2005) “Grand Challenge 2005,” DARPA http://www.grandchallenge.org 